\title{
Elementary proofs of two theorems involving arguments of eigenvalues of a product of two unitary matrices
}

\author{
Hoi Fung Chau ${ }^{1,2^{*}}$ and Yan Ting Lam ${ }^{1,3}$
}

* Correspondence: hfchau@hku.hk ${ }^{1}$ Department of Physics, University of Hong Kong, Pokfulam Road, Hong Kong

Full list of author information is available at the end of the article

\section{Abstract}

We give elementary proofs of two theorems concerning bounds on the maximum argument of the eigenvalues of a product of two unitary matrices-one by Childs et al. [J. Mod. Phys. 47, 155 (2000)] and the other one by Chau [Quant. Inf. Comp. 11, 721 (2011)]. Our proofs have the advantages that the necessary and sufficient conditions for equalities are apparent and that they can be readily generalized to the case of infinite-dimensional unitary operators.

Let $\operatorname{Eig}(U)$ denotes the set of all eigenvalues of a unitary matrix $U$. Interestingly, one can give non-trivial information on $\operatorname{Eig}(U V)$, usually in the form of inequalities, solely based on $\operatorname{Eig}(U)$ and $\operatorname{Eig}(V)$. (See, for example, Refs. [1,2] for comprehensive reviews of the field of spectral variation theory of matrices, including Hermitian and normal ones.) In this paper, we give elementary proofs of two such inequalities. Let us begin by introducing a few notations first.

Definition 1. Let $U$ be a n-dimensional unitary matrix. Generalizing the conventions adopted in Ref. [2], we denote the arguments (all arguments in this paper are in principal values) of the eigenvalues of $U$ arranged in descending and ascending orders by $\theta_{j}^{\uparrow}(U)^{\prime}$ sand $\theta_{j}^{\uparrow}(U)^{\prime}$ s, respectively, where the index $j$ runs from 1 to $n$. That is to say, $\theta_{j}^{\downarrow}(U) \in(-\pi, \pi]$ where $\theta_{j}^{\downarrow}(U) \in(-\pi, \pi]$ and $\left|\phi_{j}^{\downarrow}(U)\right\rangle$ is a normalized eigenvector of $U$

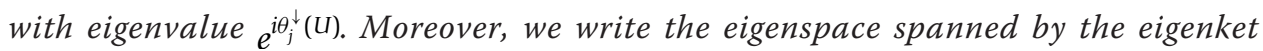
$\mathcal{H}_{j}^{\downarrow}(U)$ by $\mathcal{H}_{j}^{\downarrow}(U)$ and the eigenspace corresponding to the eigenvalue $e^{i \theta_{1}^{\downarrow}(U) b y} \mathcal{H}^{\bullet}(U)$, respectively. (Clearly, $\mathcal{H}^{\bullet}(U)=\mathcal{H}_{1}^{\downarrow}(U)$ if and only if $e^{i \theta_{1}^{\downarrow}(U) i s}$ a non-degenerate eigenvalue.) We further denote the absolute value of the argument of the eigenvalues of $U$ arranged in descending order by $|\theta|_{j}^{\downarrow}(U)^{\prime}$ s, where the index $j$ runs from 1 to $n$.

Recently, Childs et al. [3] proved the validity of the following theorem using BakerCampbell-Hausdorff formula and eigenvalue perturbation theory.

Theorem 1. Let $U, V$ be two n-dimensional unitary matrices satisfying $\theta_{1}^{\uparrow}(U)+\theta_{1}^{\uparrow}(V)>-\pi$ and $\theta_{1}^{\uparrow}(U)+\theta_{1}^{\uparrow}(V)>-\pi$. Then,

$$
\theta_{1}^{\downarrow}(U V) \leq \theta_{1}^{\downarrow}(U)+\theta_{1}^{\downarrow}(V)
$$

\section{SpringerOpen ${ }^{\circ}$}

(c) 2011 Chau and Lam; licensee Springer. This is an Open Access article distributed under the terms of the Creative Commons Attribution License (http://creativecommons.org/licenses/by/2.0), which permits unrestricted use, distribution, and reproduction in any medium, provided the original work is properly cited. 
and

$$
\theta_{1}^{\uparrow}(U V) \geq \theta_{1}^{\uparrow}(U)+\theta_{1}^{\uparrow}(V) .
$$

Furthermore, the equality of Equation $1 a$ holds if and only if $\operatorname{dim}\left[\mathcal{H}^{\bullet}(U) \cap \mathcal{H}^{\bullet}(V)\right] \geq 1$. Similarly, the equality of Equation $1 b$ holds if and only if $\operatorname{dim}\left[\mathcal{H}^{\bullet}\left(U^{-1}\right) \cap \mathcal{H}^{\bullet}\left(V^{-1}\right)\right] \geq 1$.

Actually, a more general version of Theorem 1 was first proven by Nudel'man and Švarcman [4] by looking into the geometric properties of certain hyperplanes related to the argument of the eigenvalues of a unitary matrix. Built on this geometric approach, Thompson [5] extended Nudel'man and Švarcman's result by giving an even more general version of Theorem 1. (Note that Nidel'man and Švarcman as well as Thompson used a different convention in which all arguments of the eigenvalues are taken from the interval $[0,2 \pi)$. Nonetheless, the convention does not affect the conclusions of Theorem 1.) Later on, Agnihotri and Woodward [6] as well as Biswas [7] showed among other things the validity of Theorem 1 by means of quantum Schubert calculus. Belkale [8] obtained Theorem 1 by studying the local monodromy of certain geometrical objects.

Along a similar line of investigation, Chau [9] recently showed among other things the following theorem using Rayleigh-Schrödinger series.

Theorem 2. Let $U, V$ be two n-dimensional unitary matrices. Then,

$$
|\theta|_{1}^{\downarrow}(U V) \leq|\theta|_{1}^{\downarrow}(U)+|\theta|_{1}^{\downarrow}(V) .
$$

Moreover, the equality holds if and only if

$$
\begin{aligned}
& \text { 1. }|\theta|_{1}^{\downarrow}(U)+|\theta|_{1}^{\downarrow}(V) \leq \pi \text {, and } \\
& \text { 2. (a) } \operatorname{dim}\left[\mathcal{H}^{\bullet}(U) \cap \mathcal{H}^{\bullet}(V)\right] \geq 1, \theta_{1}^{\downarrow}(U)=|\theta|_{1}^{\downarrow}(U) \text { and } \theta_{1}^{\downarrow}(V)=|\theta|_{1}^{\downarrow}(V) \text {; or } \\
& \text { (b) } \operatorname{dim}\left[\mathcal{H}^{\bullet}\left(U^{-1}\right) \cap \mathcal{H}^{\bullet}\left(V^{-1}\right)\right] \geq 1, \theta_{1}^{\uparrow}(U)=-|\theta|_{1}^{\downarrow}(U) \text { and } \theta_{1}^{\uparrow}(V)=-|\theta|_{1}^{\downarrow}(V) \text {. }
\end{aligned}
$$

Note that all existing proofs of Theorems 1 and 2 involve rather high level geometrical or analytical methods. Here, we report elementary proofs of these two theorems. One of the advantages of these elementary proofs is that one can easily deduce the necessary and sufficient conditions for equalities. Besides, it is straightforward to extend the theorem to cover the case of infinite-dimensional unitary operators.

Our elementary proofs of these two theorems rely on Lemma 2, which in turn follows from Lemma 1.

Lemma 1. Let $U, V$ be two n-dimensional unitary matrices with $\theta_{1}^{\downarrow}(U)-\theta_{1}^{\uparrow}(U)$, $\theta_{1}^{\downarrow}(U)+\theta_{1}^{\downarrow}(V), \theta_{1}^{\downarrow}(U)+\theta_{1}^{\downarrow}(V),-\theta_{1}^{\uparrow}(U)-\theta_{1}^{\uparrow}(V)<\pi$. Then,

$$
\arg \left\langle\phi_{j}^{\downarrow}(U V)|U| \phi_{j}^{\downarrow}(U V)\right\rangle+\arg \left\langle\phi_{j}^{\downarrow}(U V)|V| \phi_{j}^{\downarrow}(U V)\right\rangle=\theta_{j}^{\downarrow}(U V)
$$

for $j=1,2, \ldots, n$.

Proof. By definition, $U V\left|\phi_{j}^{\downarrow}(U V)\right\rangle=e^{i \theta_{j}^{\downarrow}(U V)}\left|\phi_{j}^{\downarrow}(U V)\right\rangle$. Since $U$ is unitary, we know that $\left\langle\phi_{j}^{\downarrow}(U V)\left|U^{-1}\right| \phi_{j}^{\downarrow}(U V)\right\rangle=e^{i \theta_{j}^{\downarrow}(U V)}\left[\left\langle\phi_{j}^{\downarrow}(U V)|U| \phi_{j}^{\downarrow}(U V)\right\rangle\right]^{*}-$ $\left\langle\phi_{j}^{\downarrow}(U V)\left|U^{-1}\right| \phi_{j}^{\downarrow}(U V)\right\rangle=e^{i \theta_{j}^{\downarrow}(U V)}\left[\left\langle\phi_{j}^{\downarrow}(U V)|U| \phi_{j}^{\downarrow}(U V)\right\rangle\right]^{*}$. By taking the arguments in both sides, we obtain 


$$
\arg \left\langle\phi_{j}^{\downarrow}(U V)|V| \phi_{j}^{\downarrow}(U V)\right\rangle=\theta_{j}^{\downarrow}(U V)-\arg \left\langle\phi_{j}^{\downarrow}(U V)|U| \phi_{j}^{\downarrow}(U V)\right\rangle \bmod 2 \pi .
$$

Note that for any normalized state ket $|\psi\rangle,\langle\psi|U| \psi\rangle$ and $\langle\psi|V| \psi\rangle$ are located in the convex hull formed by the vertices $\left\{e^{i \theta_{k}^{\downarrow}(U)}\right\}_{k=1}^{n}$ and $\left\{e^{i \theta_{k}^{\downarrow}(V)}\right\}_{k=1}^{n}$ on the complex plane $\mathbb{C}$, respectively. Combined with the conditions that $\theta_{1}^{\downarrow}(U)-\theta_{1}^{\uparrow}(U), \theta_{1}^{\downarrow}(V)-\theta_{1}^{\uparrow}(V)<\pi$, we have $\quad \arg \left\langle\phi_{j}^{\downarrow}(U V)|U| \phi_{j}^{\downarrow}(U V)\right\rangle \in\left[\theta_{1}^{\uparrow}(U), \theta_{1}^{\downarrow}(U)\right] \quad$ and $\arg \left\langle\phi_{j}^{\downarrow}(U V)|V| \phi_{j}^{\downarrow}(U V)\right\rangle \in\left[\theta_{1}^{\uparrow}(V), \theta_{1}^{\downarrow}(V)\right]$. Since $\theta_{1}^{\downarrow}(U)+\theta_{1}^{\downarrow}(V),-\theta_{1}^{\uparrow}(U)-\theta_{1}^{\uparrow}(V)<\pi$, we conclude that Equation 4 is valid even if the modulo $2 \pi$ is removed. $\square$

Lemma 2. Let $U$ be a n-dimensional unitary matrix with $\theta_{1}^{\downarrow}(U)-\theta_{1}^{\uparrow}(U)<\pi$. Then, for $j=1,2, \ldots, n$, we have

$$
\theta_{j}^{\downarrow}(U)=\min _{\mathcal{H}: \operatorname{codim} \mathcal{H}=j-1} \max _{|\psi\rangle \in \mathcal{H}} \arg \langle\psi|U| \psi\rangle .
$$

Furthermore, the extremum in the R.H.S. of the above equation is attained by choosing $\mathcal{H}=\oplus_{k=j}^{n} \mathcal{H}_{k}^{\downarrow}(U)$. In particular,

$$
\theta_{1}^{\uparrow}(U) \leq \arg \langle\psi|U| \psi\rangle \leq \theta_{1}^{\downarrow}(U)
$$

for all $|\psi\rangle$.

Proof. Any Hilbert subspace of codimension $j-1$ must have non-trivial intersection with the $j$-dimensional Hilbert space $\oplus_{k=1}^{j} \mathcal{H}_{k}^{\downarrow}(U)$. In addition, the set $S=\left\{\langle\psi|U| \psi\rangle:|\psi\rangle \in \oplus_{k=1}^{j} \mathcal{H}_{k}^{\downarrow}(U)\right.$ and $\left.\langle\psi \mid \psi\rangle=1\right\}$ is equal to the convex hull formed by the vertices $\left\{e^{i \theta_{k}^{\downarrow}(U)}\right\}_{k=1}^{j}$ on the complex plane $\mathbb{C}$. Since $\theta_{1}^{\downarrow}(U)-\theta_{j}^{\downarrow}(U) \leq \theta_{1}^{\downarrow}(U)-\theta_{1}^{\uparrow}(U)<\pi, S$ lies on a half plane on $\mathbb{C}$ and $S$ does not intersect with the negative real half line. Hence, every normalized vector $|\psi\rangle$ in $\oplus_{k=1}^{j} \mathcal{H}_{k}^{\downarrow}(U)$ must obey $\arg \langle\psi|U| \psi\rangle \geq \theta_{j}^{\downarrow}(U)$; and the equality holds if $|\psi\rangle=\left|\phi_{j}^{\downarrow}(U)\right\rangle$ up to a phase. (This condition for equality is both necessary and sufficient provided that $e^{i \theta_{j}^{\downarrow}(U)}$ is a non-degenerate eigenvalue of $U$.) Hence, the R.H.S. of Equation 5 must be greater than or equal to $\theta_{j}^{\downarrow}(U)$. On the other hand, by applying a similar convex hull argument to the codimension $j$ - 1 subspace $\mathcal{H}^{\prime}=\oplus_{k=j}^{n} \mathcal{H}_{k}^{\downarrow}(U)$, we know that $\max _{\mid \psi\} \in \mathcal{H}^{\prime}} \arg \langle\psi|U| \psi\rangle=\theta_{j}^{\downarrow}(U)$. And the maximum value is attained by picking $|\psi\rangle=\left|\phi_{j}^{\downarrow}(U)\right\rangle$. Hence, Equation 5 is true.

Lastly, we deduce the second inequality in Equation 6 by putting $j=1$ in Equation 5 . And then, we obtain the first inequality in Equation 6 by substituting $U$ by $U^{-1}$ into the second inequality. $\square$

Lemma 2 is of interest in its own right for it is analogous to the famous minmax principle for Hermitian matrices. (See, for example, Theorem 6.1 in Ref. [1].)

We now give the elementary proofs of Theorems 1 and 2 .

Elementary proof of Theorem 1. We only need to show the validity of Equation 1a as the validity of Equation $1 \mathrm{~b}$ follows directly from it. This is because $\theta_{j}^{\uparrow}\left(U^{-1}\right)=-\theta_{j}^{\downarrow}(U)$ for all $n$-dimensional unitary matrices $U$ and for $j=1,2, \ldots, n$. 
Since $\theta_{1}^{\downarrow}(U)+\theta_{1}^{\downarrow}(V) \leq \pi$ and $\theta_{1}^{\uparrow}(U)+\theta_{1}^{\uparrow}(V)>-\pi$, we have the following three cases to consider.

Case (i): $\theta_{1}^{\downarrow}(U)-\theta_{1}^{\uparrow}(U), \theta_{1}^{\downarrow}(V)-\theta_{1}^{\uparrow}(V)<\pi$;

Case (ii): $\pi \leq \theta_{1}^{\downarrow}(U)-\theta_{1}^{\uparrow}(U)<2 \pi$ and $\theta_{1}^{\downarrow}(V)-\theta_{1}^{\uparrow}(V)<\pi$;

Case (iii): $\pi \leq \theta_{1}^{\downarrow}(V)-\theta_{1}^{\uparrow}(V)<2 \pi$ and $\theta_{1}^{\downarrow}(U)-\theta_{1}^{\uparrow}(U)<\pi$.

To prove the validity of Equation 1a for case (i), we apply Lemma 1 to obtain

$$
\theta_{1}^{\downarrow}(U V)=\arg \left\langle\phi_{1}^{\downarrow}(U V)|U| \phi_{1}^{\downarrow}(U V)\right\rangle+\arg \left\langle\phi_{1}^{\downarrow}(U V)|V| \phi_{1}^{\downarrow}(U V)\right\rangle .
$$

Separately applying Equation 6 in Lemma 2 to the two terms in the R.H.S. of Equation 7 , we have

$$
\theta_{1}^{\downarrow}(U V) \leq \theta_{1}^{\downarrow}(U)+\theta_{1}^{\downarrow}(V) .
$$

Hence, Equation 1a is valid for case (i). Furthermore, the equality holds if and only if $\left|\phi_{1}^{\downarrow}(U V)\right\rangle \in \mathcal{H}^{\bullet}(U) \cap \mathcal{H}^{\bullet}(V)$. This proves the validity of this theorem for case (i).

The validity of cases (ii) and (iii) follow that of case (i). (For simplicity, we only consider the reduction from case (ii) to case (i) as the reduction from case (iii) to case (i) is similar.) Let $U, V$ be a pair of unitary matrices satisfying the conditions of case (ii). Then, $\theta_{1}^{\downarrow}(U)+\theta_{1}^{\downarrow}(V)-\theta_{1}^{\uparrow}(U)-\theta_{1}^{\uparrow}(V)<2 \pi$. So, we can pick a number $a$ from the non-empty open interval

$$
a \in\left(\frac{\theta_{1}^{\downarrow}(U)-\theta_{1}^{\uparrow}(U)-\pi}{\theta_{1}^{\downarrow}(U)-\theta_{1}^{\uparrow}(U)}, \frac{\pi-\theta_{1}^{\downarrow}(V)+\theta_{1}^{\uparrow}(V)}{\theta_{1}^{\downarrow}(U)-\theta_{1}^{\uparrow}(U)}\right) .
$$

It is easy to check that $a \in(0,1)$ and that $0<a\left[\theta_{1}^{\downarrow}(U)-\theta_{1}^{\uparrow}(U)\right]$, $a\left[\theta_{1}^{\downarrow}(U)-\theta_{1}^{\uparrow}(U)\right]+\theta_{1}^{\downarrow}(V)-\theta_{1}^{\uparrow}(V)<\pi, a\left[\theta_{1}^{\downarrow}(U)-\theta_{1}^{\uparrow}(U)\right]+\theta_{1}^{\downarrow}(V)-\theta_{1}^{\uparrow}(V)<\pi$. As a result, the pair of matrices $U^{a}$ and $V$ satisfies the conditions of this theorem for case (i) where the notation $U^{a}$ denotes the unitary matrix $\sum_{j} e^{i a \theta_{j}^{\downarrow}(U)}\left|\phi_{j}^{\downarrow}(U)\right\rangle\left\langle\phi_{j}^{\downarrow}(U)\right|$. Therefore, $\theta_{1}^{\downarrow}\left(U^{a} V\right) \leq \theta_{1}^{\downarrow}\left(U^{a}\right)+\theta_{1}^{\downarrow}(V)=a \theta_{1}^{\downarrow}(U)+\theta_{1}^{\downarrow}(V)$. Further notice that the pair of matrices $U^{1-a}$ and $U^{a} V$ also obeys the conditions of this theorem for case (i). Hence, $\theta_{1}^{\downarrow}(U V)=\theta_{1}^{\downarrow}\left(U^{1-a}\left(U^{a} V\right)\right) \leq \theta_{1}^{\downarrow}\left(U^{1-a}\right)+\theta_{1}^{\downarrow}\left(U^{a} V\right) \leq(1-a) \theta_{1}^{\downarrow}(U)+a \theta_{1}^{\downarrow}(U)+\theta_{1}^{\downarrow}(V)=\theta_{1}^{\downarrow}(U)+\theta_{1}^{\downarrow}(V)$. Clearly, for case (ii), Equation $1 \mathrm{a}$ becomes an equality if and only if $\left|\phi_{1}^{\downarrow}(U V)\right\rangle \in \mathcal{H}^{\bullet}\left(U^{1-a}\right) \cap \mathcal{H}^{\bullet}\left(U^{a} V\right) \cap \mathcal{H}^{\bullet}\left(U^{a}\right) \cap \mathcal{H}^{\bullet}(V)=\mathcal{H}^{\bullet}(U) \cap \mathcal{H}^{\bullet}(V)$. This proves the validity of this theorem for case (ii).

Elementary proof of Theorem 2. We may assume that $|\theta|_{1}^{\downarrow}(U)+|\theta|_{1}^{\downarrow}(V)<\pi$ for the theorem is trivially true otherwise. Then, from Equations $1 \mathrm{a}$ and $1 \mathrm{~b}$ in Theorem 1, we have

$$
\begin{aligned}
|\theta|_{1}^{\downarrow}(U V) & =\max \left[\theta_{1}^{\downarrow}(U V),-\theta_{1}^{\uparrow}(U V)\right] \\
& \leq \max \left[\theta_{1}^{\downarrow}(U)+\theta_{1}^{\downarrow}(V),-\theta_{1}^{\uparrow}(U)-\theta_{1}^{\uparrow}(V)\right] \\
& \leq|\theta|_{1}^{\downarrow}(U)+|\theta|_{1}^{\downarrow}(V) .
\end{aligned}
$$

Suppose $\theta_{1}^{\downarrow}(U)+\theta_{1}^{\downarrow}(V)>-\theta_{1}^{\uparrow}(U)-\theta_{1}^{\uparrow}(V)$, then the last inequality in the above equation is an equality if and only if $\theta_{1}^{\downarrow}(U)=|\theta|_{1}^{\downarrow}(U)$ and $\theta_{1}^{\downarrow}(V)=|\theta|_{1}^{\downarrow}(V)$. By the 
same argument, in the case of $\theta_{1}^{\downarrow}(U)+\theta_{1}^{\downarrow}(V)<-\theta_{1}^{\uparrow}(U)-\theta_{1}^{\uparrow}(V)$, the last inequality in the above equation is an equality if and only if $\theta_{1}^{\uparrow}(U)=-|\theta|_{1}^{\downarrow}(U)$ and $\theta_{1}^{\uparrow}(V)=-|\theta|_{1}^{\downarrow}(V)$. Applying Lemma 1 to analyze the condition for equality of the first inequality in Equation 10, we get the necessary and sufficient conditions for equality as stated in this theorem for the case of $|\theta|_{1}^{\downarrow}(U)+|\theta|_{1}^{\downarrow}(V)<\pi$. Whereas in the case of $|\theta|_{1}^{\downarrow}(U)+|\theta|_{1}^{\downarrow}(V)=\pi$, we use a similar trick in our elementary proof of Theorem 1 by choosing a real number $a \in(0,1)$ such that $|\theta|_{1}^{\downarrow}\left(U^{a}\right),|\theta|_{1}^{\downarrow}\left(U^{1-a}\right),|\theta|_{1}^{\downarrow}(V),|\theta|_{1}^{\downarrow}\left(U^{a}\right)+|\theta|_{1}^{\downarrow}(V)<\pi / 2$. Then, by analyzing the conditions for equality for Theorem 2 for the pairs of unitary matrices $U^{a}$ and $V$, we conclude that the necessary and sufficient conditions stated in this theorem are true for the case of $|\theta|_{1}^{\downarrow}(U)+|\theta|_{1}^{\downarrow}(V)=\pi$. $\square$

After simple modifications both in the theorems and our proofs, we find the infinitedimensional analogs of Theorems 1 and 2. Note that $\theta_{j}^{\downarrow}(U)^{\prime}$ s and the likes are no longer well defined for an infinite-dimensional unitary operator $U$. Nevertheless, we can still talk about sup $\arg (U)$ the supremum of the arguments of the spectrum of $U$. The symbols inf $\arg (U)$ and sup $|\arg |(U)$ can be similarly defined. We now state the extensions of Theorems 1 and 2 below.

Theorem 3. Let $U, V$ be two unitary operators acting on the same complex Hilbert space with $\sup \arg (U)+\sup \arg (V) \leq \pi$ and $\inf \arg (U)+\inf \arg (V)>-\pi$. Then,

$$
\sup \arg (U V) \leq \sup \arg (U)+\sup \arg (V)
$$

and

$$
\inf \arg (U V) \geq \inf \arg (U)+\inf \arg (V) .
$$

Moreover, the equality of Equation 11a holds if and only if there exists a sequence of eigenkets $\left\{\left|\psi_{j}\right\rangle\right\}_{j=1}^{\infty}$ of $U V$ such that $\lim _{j \rightarrow \infty} \arg \left\langle\psi_{j}|U V| \psi_{j}\right\rangle=\sup \arg (U V), \lim _{j \rightarrow \infty} \arg \left\langle\psi_{j}\right|$ $U\left|\psi_{j}\right\rangle=\sup \arg (U)$ and $\lim _{j \rightarrow \infty} \arg \left\langle\psi_{j}|V| \psi_{j}\right\rangle=\sup \arg (V)$. In a similar fashion, the equality of Equation $11 b$ holds if and only if there exists a sequence of eigenkets $\left\{\left|\psi_{j}\right\rangle\right\}_{j=1}^{\infty}$ of $U V$ such that $\lim _{j \rightarrow \infty} \arg \left\langle\psi_{j}|U V| \psi_{j}\right\rangle=\inf \arg (U V), \lim _{j \rightarrow \infty} \arg \left\langle\psi_{j}|U| \psi_{j}\right\rangle=$ $\inf \arg (U)$ and $\lim _{j \rightarrow \infty} \arg \left\langle\psi_{j}|V| \psi_{j}\right\rangle=\inf \arg (V)$.

Theorem 4. Let $U, V$ be two unitary operators acting on the same complex Hilbert space. Then,

$$
\sup |\arg |(U V) \leq \sup |\arg |(U)+\sup |\arg |(V) .
$$

Moreover, the equality holds if and only if

1. $\sup |\arg |(U)+\sup |\arg |(V) \leq \pi ;$

2. there exist a sequence of eigenkets $\left\{\left|\psi_{j}\right\rangle\right\}_{j=1}^{\infty}$ of $U V$ such that $\lim _{j \rightarrow \infty}\left|\arg \left\langle\psi_{j}|U V| \psi_{j}\right\rangle\right|$ = sup $|\arg |(U V) ;$ and

3. (a) $\lim _{j \rightarrow \infty} \arg \left\langle\psi_{j}|U| \psi_{j}\right\rangle=\sup \arg (U)=\sup |\arg |(U)$ and $\lim _{j \rightarrow \infty} \arg \left\langle\psi_{j}|V| \psi_{j}\right\rangle=$ $\sup \arg (V)=\sup |\arg |(V) ;$ or

(b) $\lim _{j \rightarrow \infty} \arg \left\langle\psi_{j}|U| \psi_{j}\right\rangle=\inf \arg (U)=-\sup |\arg |(U)$ and $\lim _{j \rightarrow \infty} \arg \left\langle\psi_{j}|V| \psi_{j}\right\rangle=$ $\inf \arg (V)=-\sup |\arg |(V)$. 
Outline proofs of Theorems 3 and 4. We can use the convex hull argument in Lemmas 1 and 2 to show that (1) $\sup \arg (U V)=\sup \arg \langle\varphi|U| \varphi\rangle+\sup \arg \langle\varphi|V| \varphi\rangle$ where the supremum is taken over all eigenkets $|\varphi\rangle$ of $U V$, and (2) inf $\arg (U) \leq \arg \langle\psi$ $|U| \psi\rangle \leq \sup \arg (U)$ for all $|\psi\rangle$ whenever sup $\arg (U)-\inf \arg (U)<\pi$. Hence, Equation 11a in Theorem 3 holds in the case of sup $\arg (U)-\inf \arg (U), \sup \arg (V)-\inf \arg (V)$ $<\pi$. Furthermore, by examining the condition for $\arg \langle\psi|U| \psi\rangle=\sup \arg (U)$ in the case of $\sup \arg (U)-\inf \arg (U)<\pi$, it is straightforward to verify the validity of the necessary and sufficient conditions for equality of Equation 11a in the case of sup arg $(U)$ - inf $\arg (U), \sup \arg (V)-\inf \arg (V)<\pi$. Now, we can follow the arguments in the proofs of the remaining cases in Theorem 1 as well as in the proof of Theorem 2 to show the validity of Theorems 3 and 4 .

\section{Acknowledgements}

We thank F.K. Chow, C.-H.F. Fung, and K.Y. Lee for their enlightening discussions. This work is supported by the RGC Grant number HKU 700709P of the HKSAR Government.

\section{Author details}

${ }^{1}$ Department of Physics, University of Hong Kong, Pokfulam Road, Hong Kong ${ }^{2}$ Center of Computational and Theoretical Physics, University of Hong Kong, Pokfulam Road, Hong Kong ${ }^{3}$ Department of Mathematics, University of Hong Kong, Hong Kong

\section{Authors' contributions}

Both authors contributed equally in this paper. They read and approved the final manuscript.

\section{Competing interests}

The authors declare that they have no competing interests.

Received: 7 December 2010 Accepted: 18 July 2011 Published: 18 July 2011

\section{References}

1. Bhatia, R: Perturbation Bounds For Matrix Eigenvalues. SIAM, Philadelphia (2007)

2. Bhatia, R: Matrix Analysis. Springer, Berlin (1997)

3. Childs, AM, Preskill, J, Renes, J: Quantum information and precision measurement. J Mod Opt. 47, 155-176 (2000)

4. Nudel'man, AA, Švarcman, PA: The spectrum of the product of unitary matrices (in Russian). Uspehi Math Nauk. 13, 111-117 (1958)

5. Thompson, RC: On the eigenvalues of a product of unitary matrices. I Lin Multilin Alg. 2, 13-24 (1974). doi:10.1080/ 03081087408817038

6. Agnihotri, S, Woodward, C: Eigenvalues of products of unitary matrices and quantum Schubert calculus. Math Res Lett. 5, 817-836 (1998)

7. Biswas, I: On the existence of unitary at connections over the punctured sphere with given local monodromy around the punctures. Asian J Math. 3, 333-344 (1999)

8. Belkale, P: Local systems on $\mathbb{P}^{1}$ - S for S a finite set. Composition Math. 129, 67-86 (2001). doi:10.1023/A:1013195625868

9. Chau, HF: Metrics on unitary matrices and their application to quantifying the degree of non-commutativity between unitary matrices. Quant Inf Comp. 11, 721-740http://arxiv.org/abs/1006.3614V2 (2011) 